\title{
Patchy echogenicity of the liver in patients with chronic hepatitis B does not indicate poorer elasticity
}

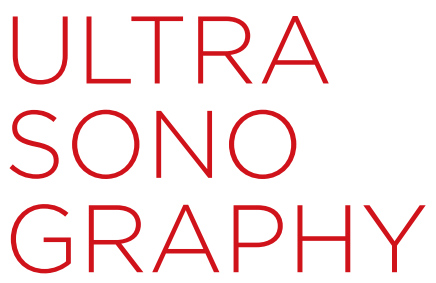

\author{
Size $\mathrm{Wu}^{1}$, Rong Tu${ }^{2}$, Xian Liang ${ }^{1}$ \\ Departments of ${ }^{1}$ Ultrasound and ${ }^{2}$ Radiology, The First Affiliated Hospital of Hainan Medical \\ University, Haikou, China
}

\section{ORIGINAL ARTICLE}

https://doi.org/10.14366/usg. 18071 pISSN: 2288-5919 - elSSN: 2288-5943 Ultrasonography 2019;38:327-335

Purpose: This study was conducted to investigate whether the presence of patchy echogenicity in the liver of patients with chronic hepatitis $B$ (CHB) is predictive of liver stiffness.

Methods: A total of $200 \mathrm{CHB}$ patients with and without patchy echogenicity of the liver were assigned to two groups, with 100 patients in each group, and 32 of them underwent liver biopsy. Additionally, 80 healthy subjects, 100 inactive hepatitis B virus (HBV) carriers, and 100 patients with decompensated hepatic cirrhosis were assigned to the control groups. Laboratory tests and clinical data were collected, and shear wave velocity (SWV) of the liver was measured for all 480 subjects.

Results: The median SWV in patients with a normal liver, inactive HBV carriers, CHB patients with and without patchy echogenicity, and decompensated hepatic cirrhosis were $1.07 \mathrm{~m} /$ $\mathrm{sec}, 1.08 \mathrm{~m} / \mathrm{sec}, 1.16 \mathrm{~m} / \mathrm{sec}, 1.16 \mathrm{~m} / \mathrm{sec}$, and $2.02 \mathrm{~m} / \mathrm{sec}$, respectively; there was no significant difference in SWV values between CHB patients with patchy echogenicity and those without patchy echogenicity. Furthermore, among CHB patients with and without patchy echogenicity, no significant difference in SWV was found according to fibrosis stage.

Conclusion: The presence of patchy echogenicity of the liver does not indicate a higher degree of liver stiffness.

Keywords: Hepatitis B, chronic; Patchy echogenicity of the liver; Point shear wave elastography; Shear wave velocity; Ultrasonography

\section{Introduction}

Hepatitis B virus (HBV) infection may result in acute and chronic hepatitis $B(C H B)$, and some patients with CHB develop liver fibrosis and cirrhosis [1]. Appropriate evaluation and treatment of CHB in its early stages may contain or mitigate the progression of liver damage and improve patients' prognosis $[1,2]$. Ultrasonography (US) plays an important role in the evaluation of chronic liver disease [3-8]. When assessed by US with a 2-4 MHz convex transducer, the livers of some patients with chronic liver disease may show minimal manifestations of abnormalities, while other livers may present typical pathological changes [3-6]. Scanning the liver with a curvilinear transducer with a frequency of $7 \mathrm{MHz}$ or above can reveal more detailed information about the liver surface and superficial

Received: November 28, 2018

Revised: February 13, 2019

Accepted: February 14, 2019

Correspondence to:

Rong Tu, PhD, MD, Department of Radiology, The First Affiliated Hospital of Hainan Medical University, No. 31, Longhua Road, Haikou 570102, China

Tel. +86-0898-66778853

Fax. +86-0898-66508833

E-mail: turong37472@126.com

\begin{abstract}
This is an Open Access article distributed under the terms of the Creative Commons Attribution NonCommercial License (http://creativecommons.org/ licenses/by-nc/4.0/) which permits unrestricted noncommercial use, distribution, and reproduction in any medium, provided the original work is properly cited.
\end{abstract}

Copyright (C) 2019 Korean Society of Ultrasound in Medicine (KSUM)

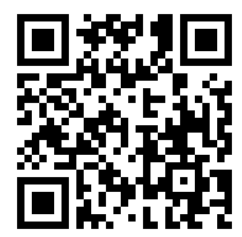

How to cite this article:

Wu S, Tu R, Liang X. Patchy echogenicity of the liver in patients with chronic hepatitis $B$ does not indicate poorer elasticity. Ultrasonography. 2019 0ct;38(4):327-335. 
parenchyma, and doing so complements routine scanning with a 2-4 MHz convex transducer $[7,8]$. However, US with a higherfrequency curvilinear transducer can also cause confusion in clinical practice, due to factors such as scattered patchy echogenicity in the liver of CHB patients. Patchy echogenicity (hyperechogenicity or both hyperechogenicity and hypoechogenicity) refers the presence of small (2-8 mm in maximal diameter), non-space-occupying, abnormal focal echogenic zones in the liver, with no detectable blood vessels using color Doppler flow imaging or power Doppler imaging. These areas show the same enhancement pattern as the adjacent liver parenchyma on contrast-enhanced US, unlike discernible nodular space-occupying lesions. These echogenic patches are usually invisible or inadequately seen on computed tomography (CT) and/ or magnetic resonance imaging (MRI) (unenhanced and contrastenhanced), and biopsy results usually show they are not tumors, dysplastic nodules, or regenerative nodules of the liver [9]. In patients with inactive HBV, CHB, or other CHB-related diseases, disease surveillance is important. Extensive research has been conducted to find optimal noninvasive approaches to evaluate liver fibrosis and cirrhosis [5,10-15]. Acoustic radiation force impulse (ARFI) point-shear-wave elastography (P-SWE) is a new approach for the evaluation of the stiffness of solid organs and soft tissue; studies have shown that shear wave velocity (SWV) can be accurately measured and that the measurements are reproducible [12-15]. Recent studies have also shown a correlation between the liver SWV measured using P-SWE and the degree of fibrosis obtained through liver biopsy $[12,16-19]$. Patchy echogenicity on the liver is a conspicuous sign suggestive of an anomaly. However, no study has investigated whether the presence of patchy echogenicity in the liver is associated with the stage of fibrosis. The objective of this study was to investigate whether liver stiffness varied between CHB patients with and without patchy echogenicity.

\section{Materials and Methods}

\section{Study Population}

This study received approval from our institutional ethics committee, and informed consent was obtained from patients. Between January 2014 and September 2018, 1,932 patients with a history of inactive HBV carrier status, CHB, or hepatitis B-related cirrhosis referred to our tertiary hospital for follow-up and US evaluation of the liver were prospectively recruited (patients referred several times were counted as a single participant). Fig. 1 presents a flowchart of sample selection. Because CHB arises from HBV infection in the normal liver, and further evolves to liver fibrosis or cirrhosis, the liver stiffness of $\mathrm{CHB}$ patients may be different from that of healthy people, inactive HBV carriers, and patients with decompensated cirrhosis. Therefore, to conduct an integrated study, we compared the liver stiffness of CHB patients with that of patients with different pathologies as controls (to determine lower and upper limit parameters of liver stiffness). Patients with decompensated cirrhosis could be diagnosed through clinical data (including laboratory tests), but patients with compensated cirrhosis could not be diagnosed accurately in the absence of biopsy and histopathology findings; therefore, patients with compensated cirrhosis were excluded. The exclusion criteria were patients with obesity (body mass index $\left.>30 \mathrm{~kg} / \mathrm{m}^{2}\right)(n=7)$, marked fatty liver $(n=10)$, pregnancy $(n=3)$, cholestasis $(n=2)$, splenectomy $(n=2)$, diabetes mellitus $(n=1)$, liver tumors ( $n=3$ ), liver nodular lesions (diameter $>7 \mathrm{~mm}$, with a spaceoccupying effect) $(n=81)$, liver congestion $(n=1)$, mitral stenosis $(n=1)$, heart failure $(n=1)$, severe chronic obstructive pulmonary disease $(n=2)$, renal failure $(n=1)$, compensated liver cirrhosis based on US criteria and biochemical tests ( $n=227)$ [20]; patients without comprehensive laboratory tests results for the evaluation of the liver ( $n=238), \mathrm{HBV}$ DNA $>2,000 \mathrm{IU} / \mathrm{mL}$, alanine transaminase (ALT) levels $>60 \mathrm{U} / \mathrm{L}$ ( $>40 \mathrm{U} / \mathrm{L}$ for inactive HBV carriers); and patients who did not agree to undergo P-SWE evaluation $(n=37)$. The inclusion criteria were referral according to the American Association for the Study of Liver Diseases guideline for the diagnosis of liver disease [1], a history of hepatitis B infection, inactive HBV, or CHB for at least 3 years, and regular follow-up since diagnosis and all inactive HBV carriers and CHB patients were inactive in recent 3 years. The diagnosis of decompensated cirrhosis was based on the presence of previous clinical data indicating a Child-Pugh class of $B$ and $C$. The study population was selected consecutively from 1,215 patients who met the inclusion criteria, including $100 \mathrm{CHB}$ patients without patchy echogenicity of the liver and $100 \mathrm{CHB}$ patients with patchy echogenicity of the liver. Among the CHB patients (with and without patchy echogenicity of the liver), 32 consecutive patients underwent percutaneous liver biopsy. One hundred inactive HBV carriers without abnormal echogenicity of the liver (HBV DNA $<1,000 \mathrm{IU} /$ $\mathrm{mL}, \mathrm{ALT}<40 \mathrm{U} / \mathrm{L}$ ) and 100 patients with decompensated liver cirrhosis were selected as control groups. Eighty participants with a healthy liver who had been hospitalized for various operations not involving the liver and did not show abnormalities on laboratory tests were recruited as a blank control group. For all patients and healthy participants, anthropometric tests were performed, including determinations of body weight, height, and body mass index. Biochemical tests of ALT, aspartate aminotransferase, $\gamma$ -glutamyltransferase, alkaline phosphatase, total bilirubin, and serum albumin levels, platelet count, and prothrombin time were performed within 7 days of the US examination. The baseline demographic and clinical characteristics of the healthy participants and patients are listed in Table 1. 
Images Acquisition

A Siemens Acuson S2000 ultrasound system (Siemens Healthcare, Ultrasound Business Unit, Mountain View, CA, USA) equipped with a curvilinear transducer $(4 \mathrm{C} 1,2-4 \mathrm{MHz}$, mean push pulse, 2.67 $\mathrm{MHz}$ ), the Virtual Touch quantification software package (version 2.0), and a $14 \mathrm{~L} 5$ linear transducer ( $6-14 \mathrm{MHz}$ ) was used to acquire US images and SWVs. A radiologist specializing in US (S.W.) with 17 years of US experience and 2 years of ARFI P-SWE experience (as of 2014) who was blinded to the clinical data performed US and P-SWE. Each US examination was first performed using the conventional B-mode. The linear transducer was used to assess the liver surface and superficial parenchyma. After the US examination of the liver parenchyma, blood vessels, and color Doppler flow imaging, P-SWE was performed. The SWV measurements were made by the same operator. P-SWE was performed with the patient in the supine position and the right arm at maximal abduction. SWV measurements of the liver were made at the $\mathrm{S} 5$ and $\mathrm{S} 6$ segments of the right liver lobe, with a depth of 1-3 cm under the liver capsule. During the procedure, patients were instructed to suspend breathing, and the acoustic beam was directed perpendicularly to the liver parenchyma free of large vessels via the intercostal space (Fig. 2). A rectangular region of interest ( $\mathrm{ROI}$ ) was set with fixed dimensions of $1.0 \mathrm{~cm} \times 0.5 \mathrm{~cm}$ with the convex transducer (4C1) when performing SWV. An adequate amount of gel was placed onto the skin overlying the liver, the transducer was placed gently onto the gel without compression, and the SWV was measured in several different ROls for each patient. Ten valid measurements were obtained at four different regions of the right lobe for each patient, and we followed the guideline that at least $60 \%$ of the measurements should be "good"; if not, a value should not be reported [21]. A "good"

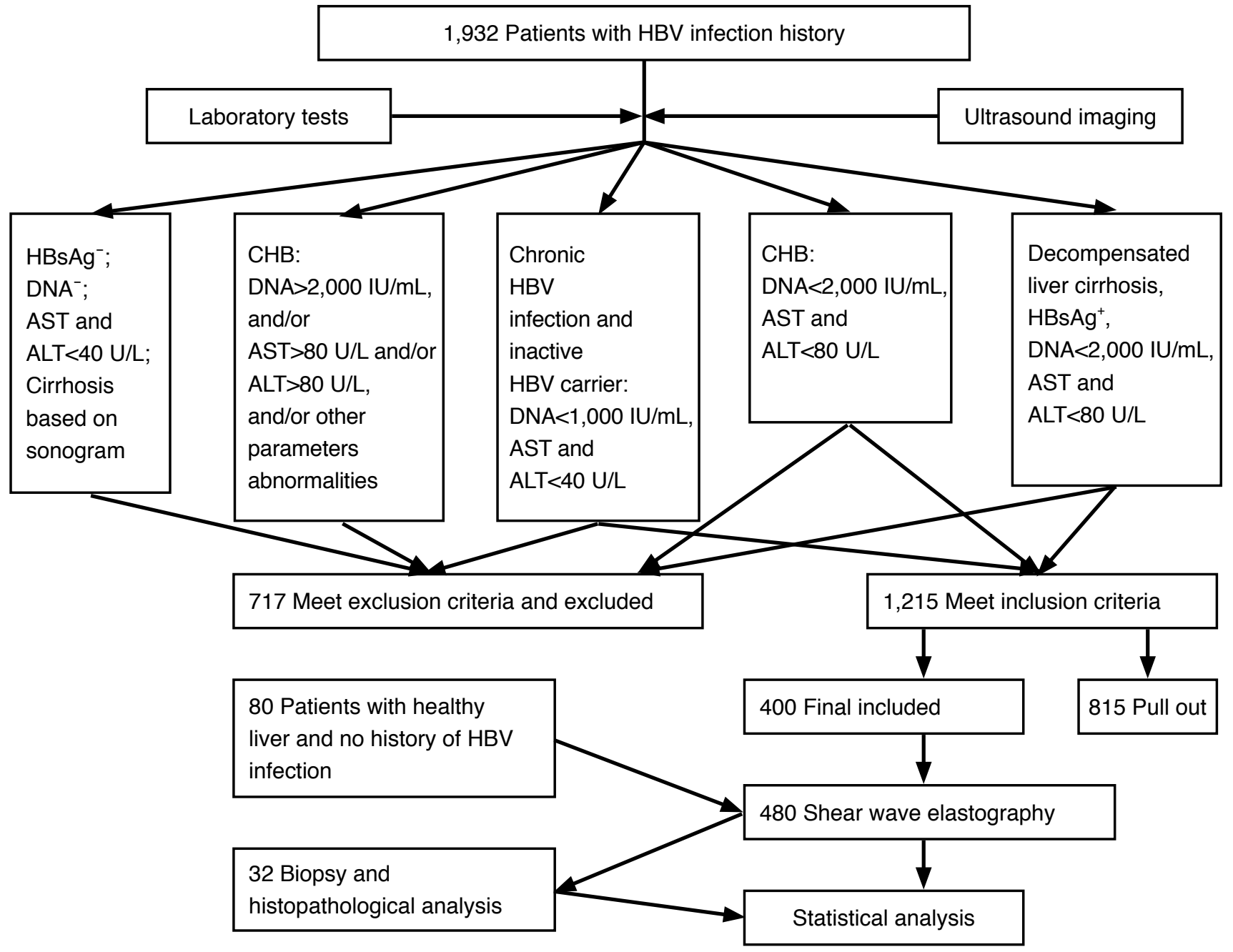

Fig. 1. Flowchart of sample selection. HBV, hepatitis B virus; HBsAg, hepatitis B surface antigen; AST, aspartate aminotransferase; ALT, alanine aminotransferase; CHB, chronic hepatitis B. 
Table 1. Baseline demographic and clinical characteristics of healthy participants and patients

\begin{tabular}{|c|c|c|c|c|c|c|}
\hline Characteristic & $\begin{array}{c}\text { Reference } \\
\text { standard }\end{array}$ & $\begin{array}{l}\text { Healthy } \\
\text { participants } \\
(n=80)\end{array}$ & $\begin{array}{c}\text { Inactive HBV } \\
\text { carriers }(n=100)\end{array}$ & $\begin{array}{l}\text { CHB without } \\
\text { patchy } \\
\text { echogenicity } \\
(n=100)\end{array}$ & $\begin{array}{l}\text { CHB with patchy } \\
\text { echogenicity } \\
(n=100)\end{array}$ & $\begin{array}{l}\text { Decompensated } \\
\text { hepatic cirrhosis } \\
\qquad(n=100)\end{array}$ \\
\hline \multicolumn{7}{|l|}{ No. of patients } \\
\hline Male & - & 46 & 47 & 51 & 53 & 55 \\
\hline Female & - & 34 & 53 & 49 & 47 & 45 \\
\hline Age (yr) & - & $33.1(25.2-44.8)$ & $39.5(33.3-52.1)$ & $40.5(33.1-53.2)$ & $39.5(33.0-53.4)$ & $48.4(41.1-57.2)$ \\
\hline Body mass index $\left(\mathrm{kg} / \mathrm{m}^{2}\right)$ & $18.5-25$ & $24.4(20.6-26.3)$ & $24.4(20.7-26.3)$ & $24.5(20.7-26.3)$ & $24.4(20.8-26.1)$ & $23.5(20.6-25.5)$ \\
\hline HBsAg & - & - & + & + & + & + \\
\hline Anti-HBsAg & + & + & - & - & - & - \\
\hline DNA of $\mathrm{HBV}(\mathrm{IU} / \mathrm{mL})$ & $0-1,000$ & 0 & $<1,000$ & $<2,000$ & $<2,000$ & $<2,000$ \\
\hline Aspartate aminotransferase (U/L) & $5-40$ & $28.6(25.0-34.0)$ & $34.9(30.2-37.9)$ & $30.8(27.1-35.5)$ & $31.1(27.1-36.1)$ & $40.4(38.5-44.2)$ \\
\hline Alanine aminotransferase (U/L) & $5-40$ & $31.2(27.1-34.4)$ & $36.2(30.63-39.1)$ & $34 . .0(29.2-38.3)$ & $33.2(28.8-36.4)$ & $37.5(35.4-39.4)$ \\
\hline Alkaline phosphatase (U/L) & $35-134$ & $79.5(65.3-102.5)$ & $89.8(65.3-117.3)$ & $87.8(70.5-115.0)$ & $87.8(70.5115 .2)$ & $87.5(73.4-117.0)$ \\
\hline Y-Glutamyl aminotransferase (U/L) & $5-54$ & $38.1(31.2-46.4)$ & $39.1(33.1-47.8)$ & $37.1(30.1-46.9)$ & $37.3(30.1-47.2)$ & $38.4(31.3-47.5)$ \\
\hline Total bilirubin $(\mu \mathrm{mol} / \mathrm{L})$ & $1.7-20$ & $8.4(5.8-10.7)$ & $8.8(5.9-10.6)$ & $8.0(5.8-10.6)$ & $8.4(5.8-10.6)$ & $8.4(5.8-10.6)$ \\
\hline Serum albumin (g/L) & $34-55$ & $44.1(38.6-49.9)$ & $44.6(40.2-48.8)$ & $45.9(40.3-49.9)$ & $42.2(40.1-49.9)$ & $36.4(33.4-39.5)$ \\
\hline Platelet count $\left(\times 10^{9} / \mathrm{L}\right)$ & $100-300$ & $198.3(138.2-234.1)$ & $206.4(139.3-246.8)$ & $206.4(138.2-249.7)$ & $206.4(138.2-246.8)$ & $198.3(149.3-216.9)$ \\
\hline Prothrombin time (sec) & $9.8-13.2$ & $10.9(10.2-11.8)$ & $11.1(9.9-11.8)$ & $10.9(9.9-11.6)$ & $10.90(9.9-11.6)$ & $12.4(11.3-13.2)$ \\
\hline
\end{tabular}

Values are median (interquartile range [25th-75th percentile]).

$H B V$, hepatitis $B$ virus; $C H B$, chronic hepatitis $B ; H B s A g$, hepatitis $B$ surface antigen.

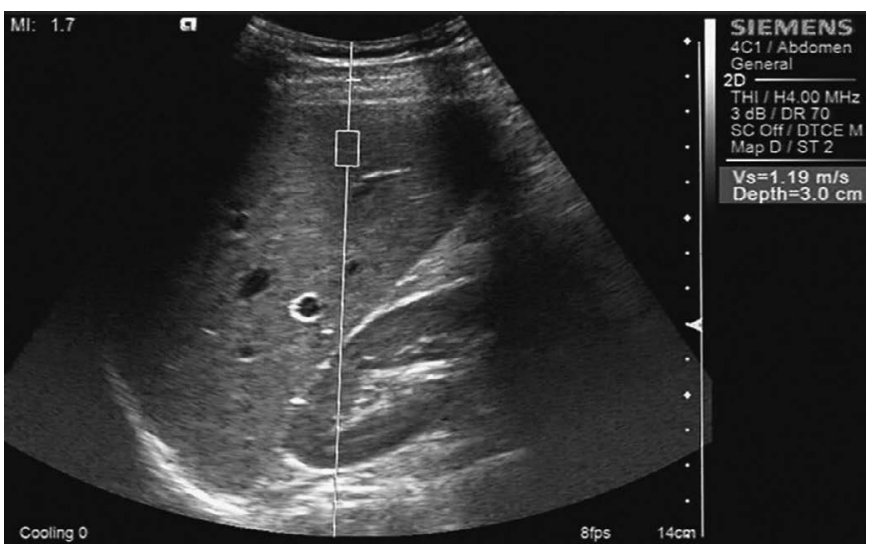

Fig. 2. Example of measurement of shear wave velocity of the liver using point shear wave elastography.

measurement was defined as one where a numerical result was obtained, not an "X.xx." The interquartile range (IQR), also called the "middle $50, "$ is a measure of the statistical dispersion defined as the difference between the upper and lower quartiles. An IQR/ median value of less than 0.30 suggests that a data set is good [21]. SWV failure was defined as a success rate of $60 \%$ or less or an IQR of $30 \%$ or more of the entire range. The median SWV was expressed in meters per second $(\mathrm{m} / \mathrm{sec})$. The mean value of all the acquisitions was calculated as the SWV result.

US, color Doppler flow imaging, and P-SWE images of the liver surface, liver parenchyma, hepatic veins, portal vein, patent paraumbilical vein (if present), and the spleen were saved in the picture archiving and communications system.

\section{Image Interpretation}

Taking normal liver and spleen parenchyma as reference areas of homogeneous moderate (not fine or coarse) parenchyma, the parenchymal texture of the participants with a healthy liver and patients with $\mathrm{CHB}$ was categorized as homogeneous moderate parenchyma, heterogeneous coarse liver parenchyma (Fig. 3), liver parenchyma without patchy echogenicity, and liver parenchyma with scattered patchy echogenicity (Figs. 4-6).

\section{Liver Histologic Assessment}

A US-guided percutaneous liver biopsy was performed within 3 days following P-SWE and US examination using a 16-gauge Magnum needle (MG1522/BARD Magnum, C.R. Bard Inc., Murray Hill, NJ, USA), and the biopsy sites were predominantly in parenchymal areas of the right lobe of the liver with scattered patchy echogenicity, and in other places in a few cases. The biopsy specimens (15-22 mm long) were fixed in formalin and embedded in paraffin. 
All biopsy specimens were analyzed by two experienced liver pathologists, who were blinded to the results of P-SWE and US, but not to the clinical and biochemical data of the patient. Liver fibrosis was evaluated using the METAVIR scoring system, as follows: score of $\mathrm{F0}$, no fibrosis; $F$ 1, portal fibrosis without septa; $F 2$, portal fibrosis and few septa; F3, numerous septa without cirrhosis; and F4,

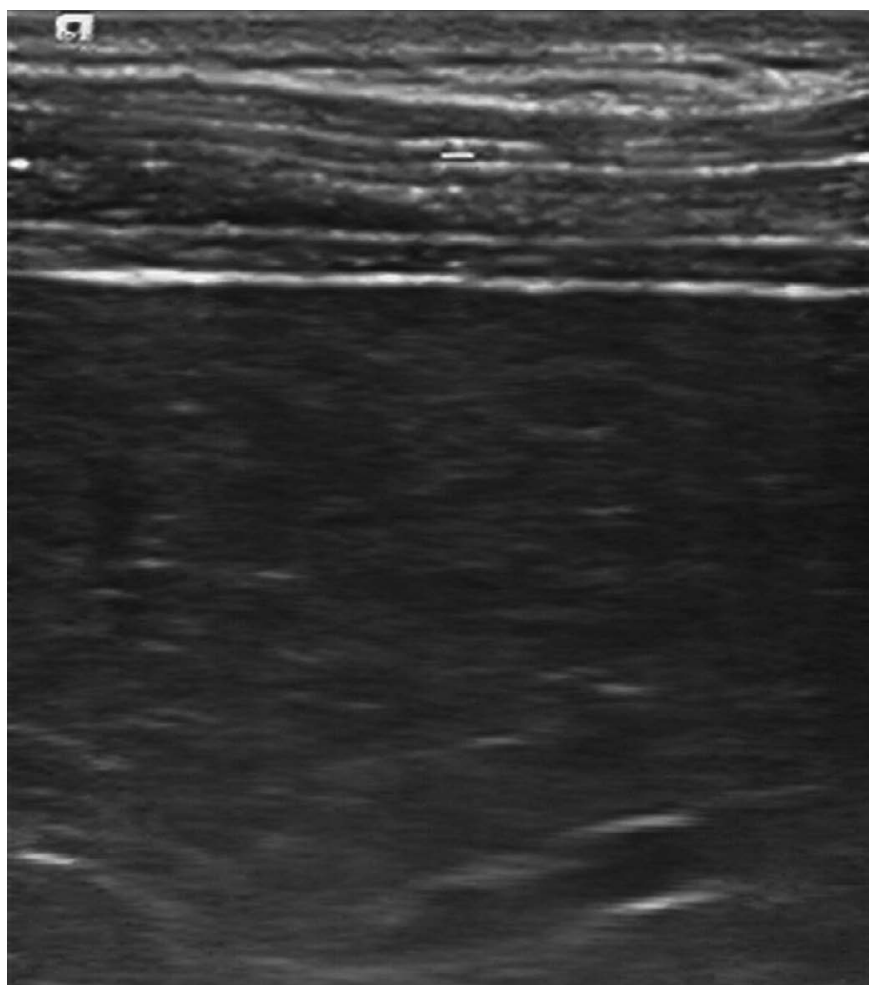

Fig. 3. A 64-year-old man with chronic hepatitis B. High-frequency ultrasonography shows heterogeneous coarse liver parenchyma.

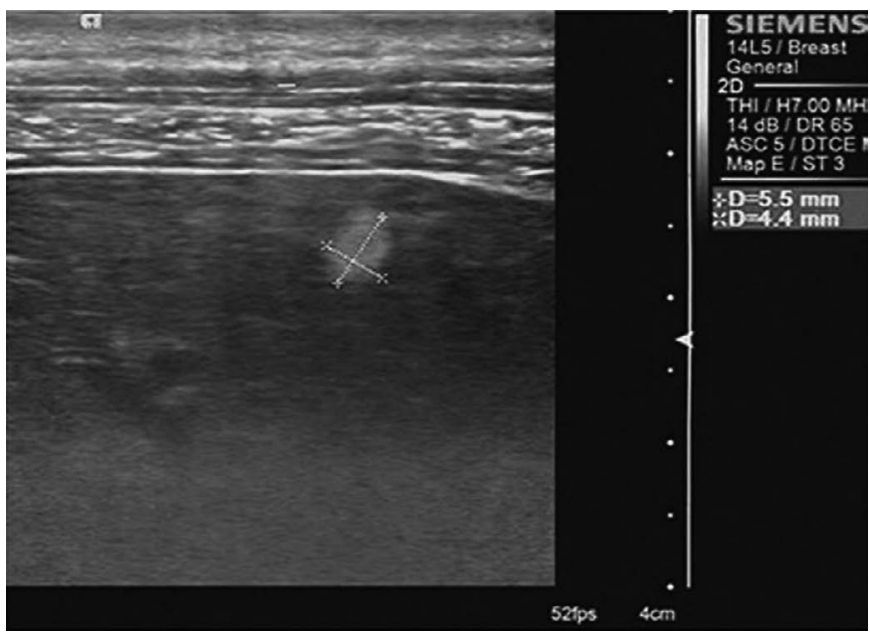

Fig. 4. A 34-year-old man with chronic hepatitis B. High-frequency ultrasonography shows coarse and patchy hyperechogenicity (cursors) in the liver parenchyma. cirrhosis. Significant fibrosis was defined as a score of $\mathrm{F} 2$ or greater [22]. Hepatic steatosis was defined as at least $30 \%$ of hepatocytes containing lipid droplets.

\section{Statistical Analysis}

When the quantitative variables were normally distributed, the results were expressed as mean values and standard deviations; otherwise, medians and IQR (25th-75th percentile) were reported. Qualitative variables were summarized as counts and percentages. The fibrosis score (METAVIR) of CHB patients with and without patchy echogenicity was compared using the chi-square test. The SWVs of the P-SWE results were not normally distributed across different groups and different fibrosis stages; therefore, the Wilcoxon rank-sum test was used to test the equality of the median SWV measurements. The level of significance was determined using a 2 -sided test with an $\alpha$ level of 0.05 . SPSS version 20 (IBM Corp., Armonk, NY, USA) was used for all statistical analyses.

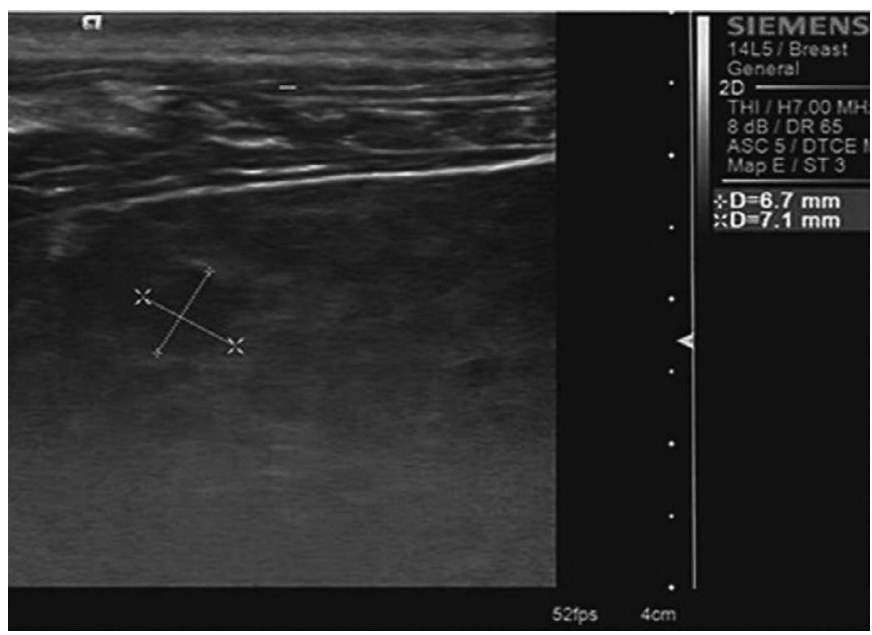

Fig. 5. A 41-year-old woman with chronic hepatitis B. High-frequency ultrasonography shows coarse and patchy hyperechogenicity (cursors) in the liver parenchyma.

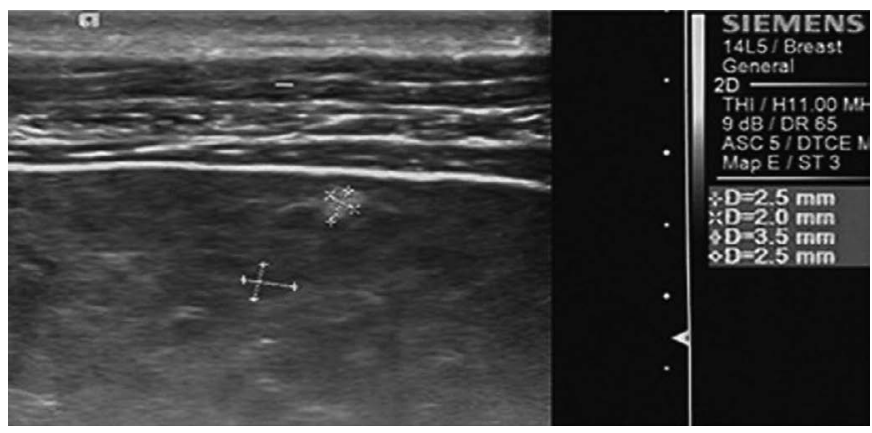

Fig. 6. A 53-year-old man with chronic hepatitis B. High-frequency ultrasonography shows coarse, patchy hyperechogenicity and hypoechogenicity (cursors) in the liver parenchyma. 


\section{Results}

The median and maximum SWV values of patients with a normal liver, inactive HBV carriers, CHB patients with and without patchy echogenicity, and patients with decompensated hepatic cirrhosis were $1.07 \mathrm{~m} / \mathrm{sec}$ and $1.26 \mathrm{~m} / \mathrm{sec}, 1.08 \mathrm{~m} / \mathrm{sec}$ and $1.41 \mathrm{~m} / \mathrm{sec}, 1.16$ $\mathrm{m} / \mathrm{sec}$ and $1.46 \mathrm{~m} / \mathrm{sec}, 1.16 \mathrm{~m} / \mathrm{sec}$ and $1.45 \mathrm{~m} / \mathrm{sec}$, and $2.02 \mathrm{~m} /$ sec and $4.17 \mathrm{~m} / \mathrm{sec}$, respectively. There was no significant difference between patients with a normal liver and inactive HBV carriers $(P=0.195)$, or between CHB patients with patchy echogenicity and those without patchy echogenicity $(P=0.354)$. However, there were significant differences between all other groups (all $\mathrm{P}<0.001$ ) (Table 2). Among the CHB patients with and without patchy echogenicity who underwent biopsy, the distribution of fibrosis scores (METAVIR: F0, F1, F2, F3, and F4) was $(0,6,7,3$, and 3$)$ and $(0,4,5,3$, and 2$)$, respectively. There was no significant difference in SWV among CHB patients according to fibrosis stage (all $P>0.99$ ). A comparison of SWVs between patients with CHB with and without patchy echogenicity did not show a significant difference $(P=0.565)$. A comparison of SWVs between CHB patients with and without patchy echogenicity according to METAVIR fibrosis stage ( $F 0-F 4)$ showed no significant differences $(P>0.05)$. However, a comparison of SWVs between CHB patients (with and without patchy echogenicity) between different fibrosis stages showed a mixed pattern of statistical significance: SWV of F1 vs. F2, $P=0.063$; $F 2$ vs. $F 3, P<0.001 ; F 3$ vs. $F 4, P=0.636$; and $F 1$ and $F 2$ vs. $F 3$ and $F 4, P<0.001$ (Table 3). The pathologic results showed that 1 CHB patient with fibrosis stage $\mathrm{F} 2$ had hepatic steatosis.

Table 2. Comparisons of SWV between patients and healthy participants

\begin{tabular}{llll}
\hline \multirow{2}{*}{ Subject } & \multicolumn{2}{c}{$\operatorname{SWV}(\mathrm{m} / \mathrm{sec})$} & \multirow{2}{*}{ P-value } \\
\cline { 2 - 3 } & Median (IQR) & Min-Max & \\
\hline Participant & & 0.195 \\
Healthy participants $(\mathrm{n}=80)$ & $1.07(0.97-1.16)$ & $0.85-1.26^{\mathrm{a}}$ & \\
$\begin{array}{l}\text { Inactive HBV infection }(\mathrm{n}=100) \\
\text { CHB patients }\end{array}$ & $1.08(1.02-1.15)$ & $0.89-1.41^{\mathrm{b}}$ & \\
$\begin{array}{l}\text { Without patchy echogenicity } \\
\text { ( } \mathrm{n}=100,14 \text { biopsies) }\end{array}$ & $1.16(1.06-1.24)$ & $0.98-1.45^{\mathrm{c}}$ & \\
$\begin{array}{l}\text { With patchy echogenicity } \\
\text { ( } \mathrm{n}=100,18 \text { biopsies) }\end{array}$ & $1.16(1.08-1.25)$ & $0.98-1.46^{\mathrm{d}}$ & \\
$\begin{array}{l}\text { Decompensated hepatic cirrhosis } \\
\text { (n=100) }\end{array}$ & & & \\
\hline
\end{tabular}

SWV, shear wave velocity; IQR, interquartile range (25th-75th percentile); HBV, hepatitis B virus; CHB, chronic hepatitis B.

\section{Discussion}

The pathophysiology of hepatitis B-related cirrhosis is a diffuse process, characterized by fibrosis and the conversion of normal liver architecture into structurally abnormal nodules [2]. Liver fibrosis is defined as an abnormal increase in collagen deposition and other components of the extracellular matrix in response to chronic injury [2].

Changes in liver stiffness are an important characteristic of pathological changes in the liver $[12,21]$. In this study, the SWVs of CHB patients with and without patchy echogenicity were significantly higher than those of patients with a normal liver and inactive HBV carriers, and lower than that of patients with decompensated cirrhosis. This finding indicates that patchy echogenicity is significantly associated with hepatic pathological progression, and the more severe the chronic inflammation and liver damage, the stiffer the liver. However, the SWVs and fibrosis stages did not show significant differences between CHB patents with patchy echogenicity and those without patchy echogenicity. This finding indicates that the degree of liver stiffness was minimally associated with the absence or presence of patchy echogenicity of the liver in patients undergoing similar pathological processes. In this study, the liver stiffness, as measured by the median SWV, ranged from $1.07 \mathrm{~m} / \mathrm{sec}$ in patients with a normal liver to $2.02 \mathrm{~m} /$ sec in patients with decompensated cirrhosis. Among CHB patients with and without patchy echogenicity, the patients in each fibrosis stage had different SWV values; there were significant differences for $F 2$ versus $F 3$ and $F 1$ and $F 2$ versus $F 3$ and $F 4$, but there were no significant differences for $F 1$ versus $F 2$ and $F 3$ versus $F 4$. These results indicate that the median liver stiffness generally increased

Table 3. Comparisons of SWV between CHB patients with and without patchy echogenicity (biopsy and histopathologically confirmed cases)

\begin{tabular}{|c|c|c|c|}
\hline \multirow{2}{*}{$\begin{array}{c}\text { Fibrosis score } \\
\text { (METAVIR) }\end{array}$} & \multicolumn{2}{|c|}{$\mathrm{SWV}(\mathrm{m} / \mathrm{sec})$} & \multirow[b]{2}{*}{ P-value } \\
\hline & $\begin{array}{l}\text { CHB without patchy } \\
\text { echogenicity }(n=14)\end{array}$ & $\begin{array}{c}\text { CHB with patchy } \\
\text { echogenicity }(n=18)\end{array}$ & \\
\hline F0 & - & - & - \\
\hline$F 1(n=10)$ & $1.08,1.13,1.17,1.20$ & $\begin{array}{l}1.08,1.09,1.16,1.20 \\
1.21,1.23\end{array}$ & 0.610 \\
\hline$F 2(n=11)$ & $\begin{array}{l}1.18,1.21,1.23,1.25 \\
1.27\end{array}$ & $\begin{array}{l}1.09,1.15,1.18,1.19, \\
1.22,1.26\end{array}$ & 0.195 \\
\hline F3 $(n=6)$ & $1.32,1.36,1.44$ & $1.32,1.39,1.40$ & $>0.99$ \\
\hline$F 4(n=5)$ & $1.39,1.40$ & $1.33,1.38,1.43$ & 0.800 \\
\hline
\end{tabular}

SWV of CHB without patchy echogenicity vs. SWV of CHB with patchy echogenicity, $P=0.800$; SWV of F1 vs. $F 2, P=0.063$; SWV of $F 2$ vs. $F 3, P<0.001$; SWV of $F 3$ vs. $F 4$, $P=0.636$; SWV of $F 1$ and $F 2$ vs. SWV of $F 3$ and $F 4, P<0.001$.

SWV, shear wave velocity; $C H B$, chronic hepatitis B. 
with the severity of fibrosis (F0-F4), but between immediately adjacent stages there were no significant differences, except for F2 versus F3. This observation suggests that SWV measurements cannot differentiate fine differences between different fibrosis stages, which is similar to the results presented by Jain et al. [23], but is inconsistent with the study by Zheng et al. [5], who found patients in each fibrosis stage to have significantly higher median liver stiffness than those with less fibrosis, as well as similar findings reported by Ozkan et al. [12] and Tsukano et al. [24]. The SWV of the liver showed no significant difference between patients with a normal liver and inactive HBV carriers, suggesting that if inactive HBV carriers do not suffer continuous or repeated active inflammation, their livers are likely to generate slight stiffness (with minimal to no fibrosis). This suggests that regular follow-up is important for inactive HBV carriers, and that if levels of HBV DNA, $A L T$, and related parameters remain within the normal reference range, the likelihood of progression to fibrosis may be very low.

In this study, the median, interquartile, mean, and upper and lower limits of the SWV of normal livers were found to be $1.07 \mathrm{~m} /$ $\mathrm{sec}, 0.97-1.16 \mathrm{~m} / \mathrm{sec}$, and $0.85-1.26 \mathrm{~m} / \mathrm{sec}$, respectively; these values are similar to those reported by Liao et al. [25], who found that the $95 \%$ confidence intervals of the median and mean SWV of normal livers measured at $\$ 5 / 8$ were $0.81-1.27$ and $0.82-1.27$ $\mathrm{m} / \mathrm{sec}$, respectively. However, our findings are inconsistent with the result of Hu et al. [26] that the mean SWV of normal liver was $1.31 \pm 0.25 \mathrm{~m} / \mathrm{sec}$. These discrepancies suggest that measurement variation and other factors may affect measured SWV values $[14,25]$.

In this study, only $16.7 \%$ (2 of 12) of patients with liver patchy echogenicity had cirrhosis (F4), which did not correspond to a significant difference from the proportion of patients without liver patchy echogenicity who had cirrhosis $(11.1 \%, 1$ of 9$)$; this observation shows that the presence of patchy liver echogenicity in patients with CHB is minimally related to the diagnosis of cirrhosis. Meanwhile, the median SWV of patients with biopsyconfirmed cirrhosis was found to be lower than that of patients with decompensated cirrhosis $(1.39 \mathrm{~m} / \mathrm{sec}$ vs. $2.02 \mathrm{~m} / \mathrm{sec})$, indicating that different stages of liver cirrhosis are associated with different levels of liver stiffness. Stiffness of the liver, which is thought to be mainly caused by fibrosis, could be influenced by patient-dependent factors, including liver inflammation, liver congestion, and biliary obstruction $[21,23]$; therefore, we excluded patients with certain diseases that could have affect the accuracy of SWV measurements.

Unlike on US, patchy echogenicity of the liver is usually invisible on CT and/or MRI (unenhanced and contrast-enhanced), in contrast to liver nodules, which can be detected by CT, MRI, and US. Although Buadu and Meyer [9] reported that liver focal echogenic lesions undetectable by $\mathrm{CT}$ and/or MRI were nodules, we have a different point of view on this issue. We believe that patchy echogenicity of the liver may be the residue of mild liver architectural damage or minimal regeneration, but the degree of architectural damage minimally, if at all, implicates the formation of bridging fibrous septa, and there is no diffusive disruption in the architecture of the entire liver (loss of the normal central-portal relationship). The hyperechoic patches may be the result of focal fat deposition on the sites of liver damage, or may be due to other causes.

Our study results show that SWV measurements cannot differentiate CHB patients without patchy echogenicity of the liver from CHB patients with patchy echogenicity of the liver. Although SWV measurements can help differentiate CHB patients (with or without patchy echogenicity of the liver) from patients with decompensated liver cirrhosis in general, the SWV of the liver does show overlap between CHB and decompensated liver cirrhosis, which limits the P-SWE value, as addressed by Ozkan et al. [12].

We did not include liver cirrhosis in the sample collection (excluded based on US criteria and biochemical tests, as addressed in the section describing the study population), but the biopsy results showed there were five patients with early-stage cirrhosis (F4) (15.6\%, 5 of 32), which indicates that evaluating CHB patients with or without patchy echogenicity based on the US criteria of liver cirrhosis and biochemical tests cannot rule out all cases liver cirrhosis, as also revealed by the study of Gobel et al. [27].

Our study had some limitations. The sample size of biopsied patients was small, which limited the power of this study to detect differences between fibrosis stages. The accuracy of using liver biopsies as the reference standard is limited by sampling issues, inadequate specimens, and the subjectivity of diagnosis. The other participants were predominantly assessed by clinical and laboratory tests, rather than histopathological evidence, which might compromise the overall accuracy.

In conclusion, the presence or absence of patchy echogenicity of the liver in patients with CHB revealed by high-frequency US does not indicate a higher degree of liver stiffness. In the future, we intend to further investigate whether there is a relationship between the presence of patchy echogenicity of the liver and liver stiffness (fibrosis) by performing high-frequency US, P-SWE, and 2-dimensional shear wave elastography, with validation by biopsy and histopathology.

ORCID: Size Wu: https://orcid.org/0000-0002-1086-764X; Rong Tu: https://orcid.org/00000002-0030-4376; Xian Liang: https://orcid.org/0000-0002-0175-3499

\section{Author Contributions}

Conceptualization: Wu S. Data acquisition: Wu S, Liang X. Data analysis or interpretation: Wu S. Drafting of the manuscript: Wu $S$. 
Critical revision of the manuscript: Tu R. Approval of the final version of the manuscript: all authors.

\section{Conflict of Interest}

No potential conflict of interest relevant to this article was reported.

\section{Acknowledgments}

This study was supported by Scientific research fund of Hainan provincial health and family planning commission (No.16A200100).

\section{References}

1. Terrault NA, Bzowej NH, Chang KM, Hwang JP, Jonas MM, Murad $\mathrm{MH}$, et al. AASLD guidelines for treatment of chronic hepatitis $\mathrm{B}$. Hepatology 2016;63:261-283.

2. Tacke F, Trautwein C. Mechanisms of liver fibrosis resolution. J Hepatol 2015;63:1038-1039.

3. Di Lelio A, Cestari C, Lomazzi A, Beretta L. Cirrhosis: diagnosis with sonographic study of the liver surface. Radiology 1989;172:389392.

4. Colli A, Fraquelli M, Andreoletti M, Marino B, Zuccoli E, Conte D. Severe liver fibrosis or cirrhosis: accuracy of US for detection: analysis of 300 cases. Radiology 2003;227:89-94.

5. Zheng J, Guo H, Zeng J, Huang Z, Zheng B, Ren J, et al. Twodimensional shear-wave elastography and conventional US: the optimal evaluation of liver fibrosis and cirrhosis. Radiology 2015;275:290-300.

6. Salvatore V, Borghi A, Peri E, Colecchia A, Li Bassi S, Montrone L, et al. Relationship between hepatic haemodynamics assessed by Doppler ultrasound and liver stiffness. Dig Liver Dis 2012;44:154159.

7. Nagata N, Miyachi H, Nakano A, Nanri K, Kobayashi H, Matsuzaki $S$. Sonographic evaluation of the anterior liver surface in chronic liver diseases using a 7.5-MHz annular-array transducer: correlation with laparoscopic and histopathologic findings. I Clin Ultrasound 2003;31:393-400.

8. Ferral H, Male R, Cardiel M, Munoz L, Quiroz y Ferrari F. Cirrhosis: diagnosis by liver surface analysis with high-frequency ultrasound. Gastrointest Radiol 1992;17:74-78.

9. Buadu A, Meyer MA. Small liver nodule detection with a highfrequency transducer in patients with chronic liver disease: report of 3 cases. J Ultrasound Med 2013;32:355-359.

10. Ma J, Jiang Y, Gong G. Evaluation of seven noninvasive models in staging liver fibrosis in patients with chronic hepatitis B virus infection. Eur J Gastroenterol Hepatol 2013;25:428-434.

11. Xiao G, Yang J, Yan L. Comparison of diagnostic accuracy of aspartate aminotransferase to platelet ratio index and fibrosis-4 index for detecting liver fibrosis in adult patients with chronic hepatitis B virus infection: a systemic review and meta-analysis. Hepatology 2015;61:292-302.

12. Ozkan MB, Bilgici MC, Eren E, Caltepe G, Yilmaz G, Kara C, et al. Role of point shear wave elastography in the determination of the severity of fibrosis in pediatric liver diseases with pathologic correlations. J Ultrasound Med 2017;36:2337-2344.

13. Jeong WK, Lim HK, Lee HK, Jo JM, Kim Y. Principles and clinical application of ultrasound elastography for diffuse liver disease. Ultrasonography 2014;33:149-160.

14. Samir AE, Dhyani M, Vij A, Bhan AK, Halpern EF, Mendez-Navarro $J$, et al. Shear-wave elastography for the estimation of liver fibrosis in chronic liver disease: determining accuracy and ideal site for measurement. Radiology 2015;274:888-896.

15. Hudson JM, Milot L, Parry C, Williams R, Burns PN. Inter- and intraoperator reliability and repeatability of shear wave elastography in the liver: a study in healthy volunteers. Ultrasound Med Biol 2013;39:950-955.

16. Pfeifer L, Zopf S, Siebler J, Schwitulla J, Wildner D, Wachter D, et al. Prospective evaluation of acoustic radiation force impulse (ARFI) elastography and high-frequency B-mode ultrasound in compensated patients for the diagnosis of liver fibrosis/cirrhosis in comparison to mini-laparoscopic biopsy. Ultraschall Med 2015;36:581-589.

17. Phelps A, Ramachandran R, Courtier J, Perito E, Rosenthal P, MacKenzie JD. Ultrasound elastography: is there a shear wave speed cutoff for pediatric liver fibrosis and inflammation? Clin Imaging 2017;41:95-100.

18. Perry MT, Savjani N, Bluth El, Dornelles A, Therapondos G. Point shear wave elastography in assessment of hepatic fibrosis: diagnostic accuracy in subjects with native and transplanted livers referred for percutaneous biopsy. Ultrasound Q 2016;32:201-207.

19. Gerber L, Kasper D, Fitting D, Knop V, Vermehren A, Sprinzl K, et al. Assessment of liver fibrosis with 2-D shear wave elastography in comparison to transient elastography and acoustic radiation force impulse imaging in patients with chronic liver disease. Ultrasound Med Biol 2015;41:2350-2359.

20. Chen YP, Dai L, Wang JL, Zhu YF, Feng XR, Hou JL. Model consisting of ultrasonographic and simple blood indexes accurately identify compensated hepatitis B cirrhosis. J Gastroenterol Hepatol 2008;23(8 Pt 1):1228-1234.

21. Barr RG, Ferraioli G, Palmeri ML, Goodman ZD, Garcia-Tsao G, Rubin J, et al. Elastography assessment of liver fibrosis: Society of Radiologists in Ultrasound Consensus Conference Statement. Ultrasound Q 2016;32:94-107.

22. Bedossa P, Poynard T. An algorithm for the grading of activity in chronic hepatitis C. The METAVIR Cooperative Study Group. Hepatology 1996;24:289-293.

23. Jain V, Dixit R, Chowdhury V, Puri AS, Gondal R. Can acoustic radiation force impulse elastography be a substitute for liver biopsy 
in predicting liver fibrosis? Clin Radiol 2016;71:869-875.

24. Tsukano N, Miyase S, Saeki T, Mizobe K, Iwashita H, Arima N, et al. Usefulness of virtual touch quantification for staging liver fibrosis in patients with hepatitis $C$, and factors affecting liver stiffness measurement failure compared with liver biopsy. Hepatol Res 2018;48:373-382.

25. Liao LY, Kuo KL, Chiang HS, Lin CZ, Lin YP, Lin CL. Acoustic radiation force impulse elastography of the liver in healthy patients: test location, reference range and influence of gender and body mass index. Ultrasound Med Biol 2015;41:698-704.

26. Hu Y, Gong HY, Lin HJ. Real-time tissue elastography for assessment of liver stiffness in adults without known liver disease. J Ultrasound Med 2015;34:1895-1900.

27. Gobel T, Erhardt A, Herwig M, Poremba C, Baldus SE, Sagir A, et al. High prevalence of significant liver fibrosis and cirrhosis in chronic hepatitis B patients with normal ALT in central Europe. J Med Virol 2011;83:968-973. 